\title{
Monitoring of air tunnels in a cylindrical storage device using ECT tomography
}

\author{
David Žurovec, *, Jakub Hlosta, Jan Nečas, Jiři Rozbroj, and Jiři Zegzulka \\ VŠB-Technical University of Ostrava, ENET Centre, 17.listopadu 15/2172, 70833 Ostrava-Poruba, \\ Czech Republic
}

\begin{abstract}
This article focuses on the monitoring of air tunnels in sand, which in common practice are characterised as flow disturbances in the flow of bulk materials inside storage devices, hoppers, silos etc. In this case air tunnels are monitored by the ECT (Electrical Capacitance Tomography) method. The basic principle of the ECT tomographic method is the measurement of permittivity of a measured non-conducting medium. The results gained by an ECT device are then compared with the real, precisely defined measured state. The measured results show that the monitoring of flow disturbances in storage devices using ECT is a new method which does not use optical methods during measurement.
\end{abstract}

\section{Introduction}

Industrial companies with a long history have built storage devices for bulk materials which were designed with regard to the precisely defined mechanical-physical properties of bulk materials intended for storage and associated technical processing. Modern industrial companies are forced to seek out and utilise new bulk materials which are far more advantageous for their technologies. This situation has arisen as a result of the technological and economic development of competing companies. The new bulk materials tend to have mechanical-physical properties different from the original ones, and these changes can cause disturbances during discharge from a storage device. Basic flow disturbances are easily identified by the irregularity of flow of bulk materials during discharge from storage devices. Flow irregularity may most often be caused by the formation of cavities in any place of the storage space, see Fig. 1a), arching over the discharge opening, see Fig. 1b), or central tunnel, see Fig 1c) [1, 2].

The gaining of objective information about the processes inside the storage devices is highly problematic because it is difficult to use optical monitoring methods. There do exist technologies which record the pressure characteristics of bulk materials inside storage devices, but they cannot provide information about flow faults [3, 4]. The basis for optimising each conveyor or storage device is the monitoring and identification of problem parts which do not comply with our requirements. Monitoring of the internal spaces of storage devices using ECT (Electrical Capacitance Tomography) is a modern method of gaining real information about storage processes.

\footnotetext{
* Corresponding author: david.zurovec@vsb.cz
} 


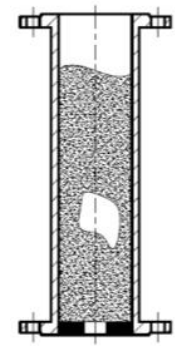

a)

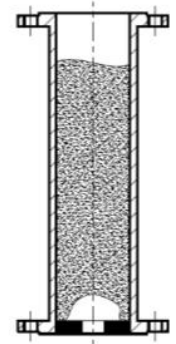

b)

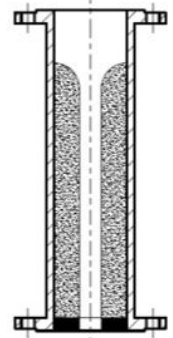

c)

Fig. 1. Basic flow disturbances of bulk materials: a) cavity, b) arch, c) tunnel.

The technology also allows us to monitor the course of filling of storage devices with bulk materials and discharge from them under various operating conditions. The ECT method provides us with results in a $2 \mathrm{D}$ graphic form through each measured cross-section and also in $3 \mathrm{D}$ depiction of the monitored space, which is defined by the dimensional interval. The 3D depiction is obtained by the interpolation of two independent $2 \mathrm{D}$ images $[5,6,7]$. The basic principle of ECT is measurement of the changes in permittivity of the measured non-conductive environment, from which information about the concentrations of the individual components in the space are obtained through calculation. A series of systematically arranged sensors is fitted in the monitored space. In our case the sensors are located around the circumference of an experimental cylindrical storage device at two planes $[3,8,9,10]$. The sensors are not in direct contact with the measured bulk material. In contrast with many other measuring systems, tomography sensors are capable of perceiving the situation through the volume at every moment and thus ensuring a dynamic image of the events within the storage device [11-15].

\section{Description of measuring system and sensor}

The measuring system consists of three basic parts, which are depicted in Fig. 2. The first part is an experimental storage device of cylindrical shape. Measuring sensors $N=12$ are fitted at two planes $(12 \times 2)$ around the circumference of the experimental storage device. Each plane measures independently and separately from the other. The individual sensors are connected to the second part, which is a tomographic device ITS $\mathrm{m} 3 \mathrm{c}$, which is a commercial ECT system supplied by the firm ITS [26]. The third part is a PC serving for the storage and processing of measured values. The detailed ECT measuring principle is described in $[12,16,17,18,19,20]$. In this article the principle is only summarized in brief. Simply put, the individual sensors located around the circumference of the experimental storage device form parallel-plate capacitors. Each pair of measuring sensors forms one parallel-plate capacitor with a certain capacitance C1-2, C1-3... C1-11, C1-12 etc., which are connected in parallel to the system. Via the individual pairs of sensors the tomographic device $\mathrm{m} 3 \mathrm{c}$ transmits regulated AC signals $\mathrm{U}[\mathrm{V}]$ to the internal space of the experimental storage device, and it then evaluates the measured change of permittivity of the nonconductive medium-dielectric. At the start the dielectric is calibrated as the "minimum, which in our case expresses the ambient air environment. The calibration of the "maximum" expresses the measured environment, which in our case is the sand. The received signals are evaluated and the result is cross-section maps of the monitored space. We identify the concentrations of both components in the monitored space. The measured data is then converted by software into a representative form on the PC. Performance parameters of the ITS m3c tomographic device are given in Table 1. 
Table 1. Performance parameters of the ITS m3c.

\begin{tabular}{|l|c|}
\hline Relative accuracy of measurement range & $0,01 \mathrm{pF}$ \\
\hline Measurement stability & $+/-0.6 \mathrm{mV}$ \\
\hline Excitation method $\boldsymbol{U}$ & $18 \mathrm{~V}$ pick-pick $\mathrm{AC}$ \\
\hline Injection frequency & $1 \mathrm{MHz}$ \\
\hline Phase shift compensation & $0^{\circ}$ to $360^{\circ}$ with $0.09^{\circ}$ resolution \\
\hline Speed of acquisition & $15 \mathrm{~ms} /$ frame \\
\hline
\end{tabular}

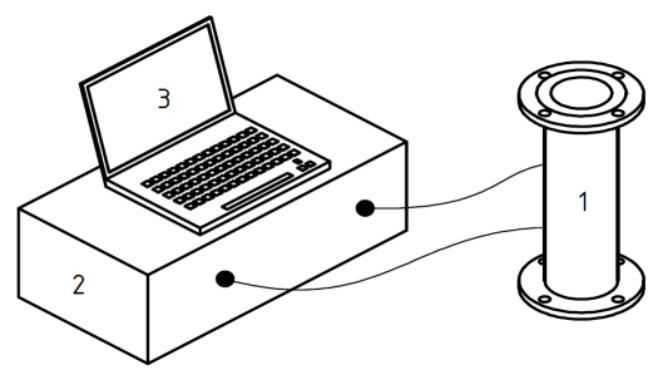

Fig. 2. Basic measuring system where: 1- Experimental storage device; 2- Tomographic device ITS $\mathrm{M} 3 \mathrm{C}$; 3- PC for evaluation of measurement.

\section{Mathematical model of tomography principle}

The capacitance of the capacitor $C[F]$ depends on the area $S\left[\mathrm{~m}^{2}\right]$ of its plates, mutual distance $r[\mathrm{~m}]$ of the plates from each other and on the permittivity of the dielectric $\varepsilon[-]$ between the plates. Generally capacitance $C[F]$ for the individual paired sensors is characterised by derivation see equation (1) to (4) and Fig. 3.

$$
E=\frac{Q}{\varepsilon \cdot S}\left[V \cdot m^{-1}\right]
$$

Where $E\left[V \cdot m^{-1}\right]$ is electric field intensity, Q[C] is electric charge, $\varepsilon[-]$ is permittivity of dielectric, $\mathrm{S}\left[\mathrm{m}^{2}\right]$ is area of the capacitor boards.

$U=\int_{(+)}^{(-)} E \cdot d r=\int_{(+)}^{(-)} \frac{Q}{\varepsilon \cdot S} \cdot d r=\frac{Q}{\varepsilon \cdot S} \int_{0}^{r} d r=\frac{Q \cdot r}{\varepsilon \cdot S}[V]$

Where $U[V]$ is capacitor voltage.

$$
C=\frac{Q}{U}=\frac{Q}{\frac{Q \cdot r}{\varepsilon \cdot S}}=\frac{Q \cdot \varepsilon \cdot S}{Q \cdot r}=\varepsilon \cdot \frac{S}{r}[F]
$$


$\varepsilon=\varepsilon_{0} \cdot \varepsilon_{r}[-]$

Where $\varepsilon_{0}[-]$ permittivity of vacuum and $\varepsilon_{\mathrm{r}}[-]$ relative permittivity of dielectrics.

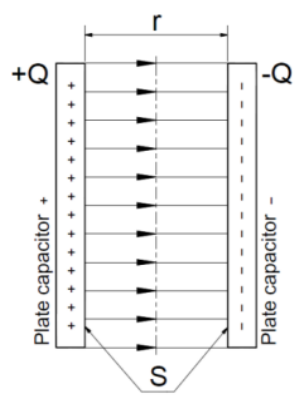

Fig. 3. Diagram of parallel-plate capacitor.

\section{Experiment and results}

The experimental measurement focuses on the monitoring of air tunnels inside a storage device filled with sand. The aim of the experiment is to determine the concentration of the individual components within the storage device, which in our case are air and sand. Thinwalled tubes with diameters of $d=\varnothing 20, \varnothing 32, \varnothing 40, \varnothing 63$ and $\varnothing 75 \mathrm{~mm}$, see Fig. 4, which in their inner space form the air tunnels that are the subject of experimental measurement, are inserted one after the other into the space of the cylindrical hopper, which has a diameter of $\mathrm{D}=100 \mathrm{~mm}$ and length of $\mathrm{L}=440 \mathrm{~mm}$. Sand is placed in the intermediate space formed by the inner cylindrical surface of the storage device and the outer cylindrical surface of the tubes. The aim is to ensure the accuracy of tomographic measurement of concentrations of the two components inside the storage device. The results of concentrations measured by the capacitance tomograph $\mathrm{m} 3 \mathrm{c}$ are compared with the real situation.
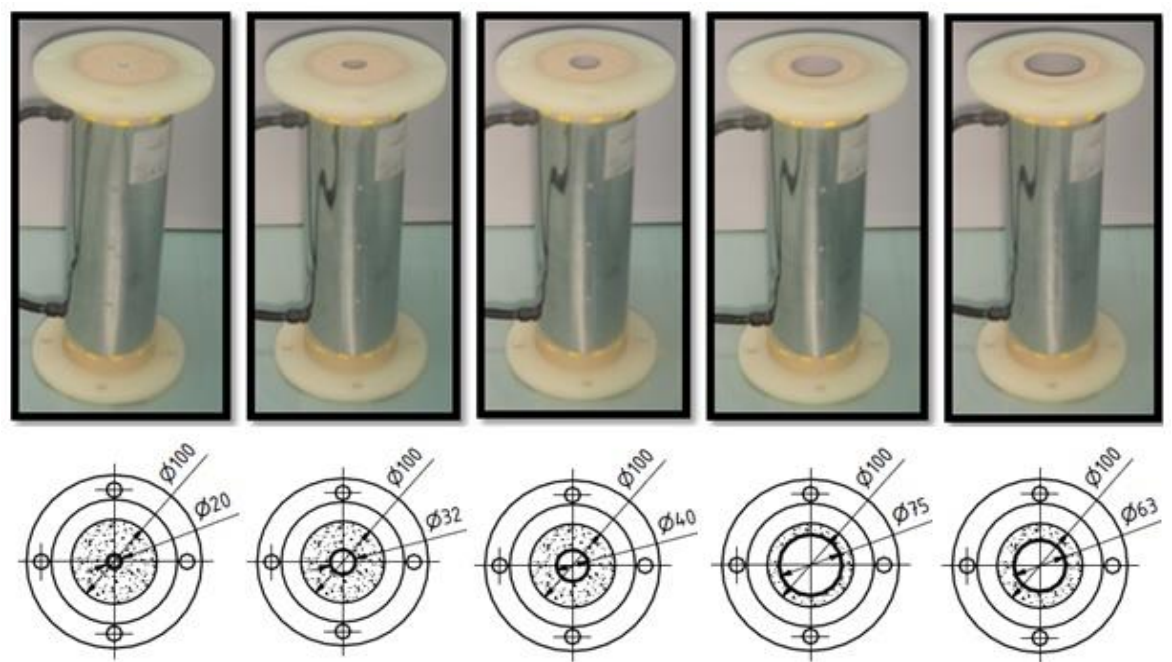

Fig. 4. Depiction of experimental storage device with air tunnel a) Ø20; b) Ø32; c) Ø40; d) Ø63; e) $\varnothing 75$. 
Characteristics of the sand, see Fig. 5, and air used for measurement:

Relative permittivity of sand " $\breve{S} H 35$ " $\varepsilon_{\mathrm{r}}=5[-]$

Relative permittivity of $\operatorname{air}_{20^{\circ} \mathrm{C}} \varepsilon_{\mathrm{r}}=1$ [-]

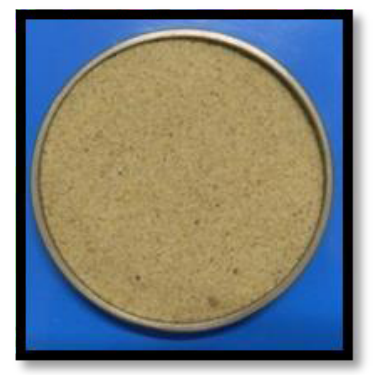

Fig. 5. Views sample of sand "ŠH 35 ".

In the context of experimental measuring by the $\mathrm{m} 3 \mathrm{c}$ capacitance tomograph the values of concentration are gained in the form of a colour depiction generated from measured concentration data. In the colour spectrum air is calibrated and depicted by the colour blue. The second monitored concentration is sand, which is calibrated as the colour red. From the resultant colour depictions of the planes P1 and P2 it is possible to read off approximately the average values of concentrations of the individual components. The precise measured values are shown in Fig. 6 . The measured values are then compared with the real situation, see Table 2, Fig. 6 and Table 3.

Table 2 contains 2D and 3D depictions of measured concentrations of sand and air. In the $2 \mathrm{D}$ cross-section one can see the air as blue, and the sand as red. The $3 \mathrm{D}$ depiction also contains the colour green, which represents the correlation transition between the air and sand. The graphic depiction of concentration in Table 2 is of a only informative nature. For example the 2D graphic depiction for Ø20 displays a $100 \%$ concentration of the sand component, but in reality a concentration was measured of $\mathrm{P} 1=96.8 \%$ and $\mathrm{P} 2=97.1 \%$, which confirms the course of concentrations in Fig. 6. The precise courses of measured concentrations of sand in the cylindrical hopper are summarised in Fig. 6. Measurements were taken for 5 different diameters of air tunnels. Each tunnel diameter was measured by the two mutually independent planes P1 and P2. A fifty concentration values were measured for each tunnel. The smaller diameters of the air tunnels display a significantly greater accuracy of measured concentrations. In contrast, for larger diameters of air tunnels relatively large deviations from the real state were measured. This uneven state is influenced by the width of the sand ring, which is at its maximum when there is the smallest diameter of air cavity, and at its minimum when there is the maximum diameter of air cavity. The width of the sand ring influences the throughput of flow waves entering the measured space. In general the permeability of current waves depends on the permittivity of the measured environment. In our case the relative permittivity of dry sand is $\varepsilon_{\text {rsand }}=5$ and the relative permittivity of air is $\varepsilon_{\text {rair }}=1$. The measured concentrations of sand given in Fig. 6 are compared with the real curves(value) of concentrations derived from the measured experiments. 
Table 2. Comparison of true and measured concentrations of sand and air at the planes P1 and P2.

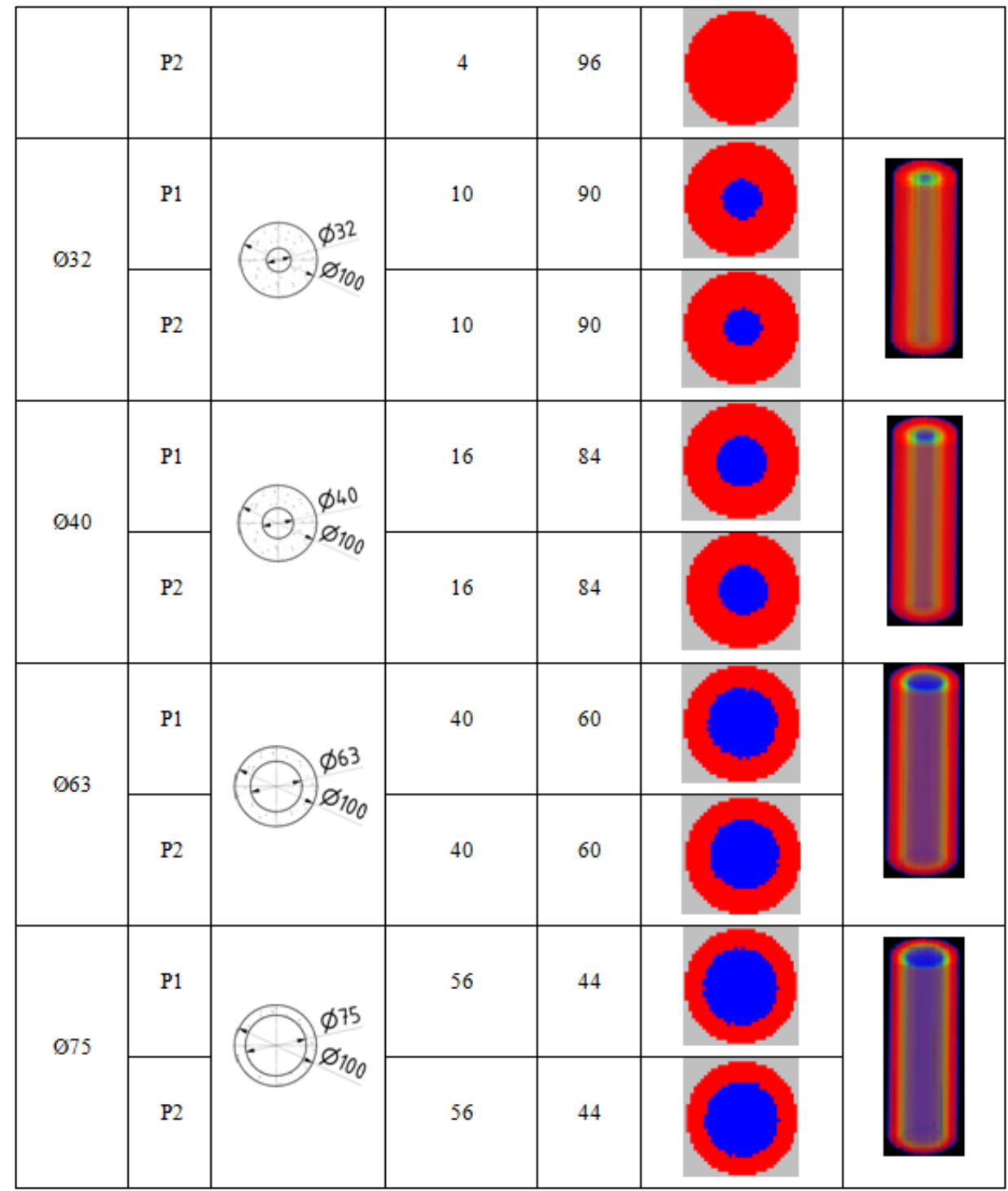

Deviations can be seen between the measured and true concentrations. The deviations of measured concentrations of sand in the cylindrical storage device are compared with the true values of concentrations based on real experimental measuring. Table 3 contains the average values of true concentrations of sand $\mathrm{C}_{\mathrm{EX}}$ based on experimental measuring and value of measured concentrations $\mathrm{C}_{\mathrm{N}}$, measured using the $\mathrm{m} 3 \mathrm{c}$ capacitance tomography. Table 3 also contains the value of absolute deviation $\Delta \mathrm{x}$ of concentrations for the individual diameters of air tunnels. 


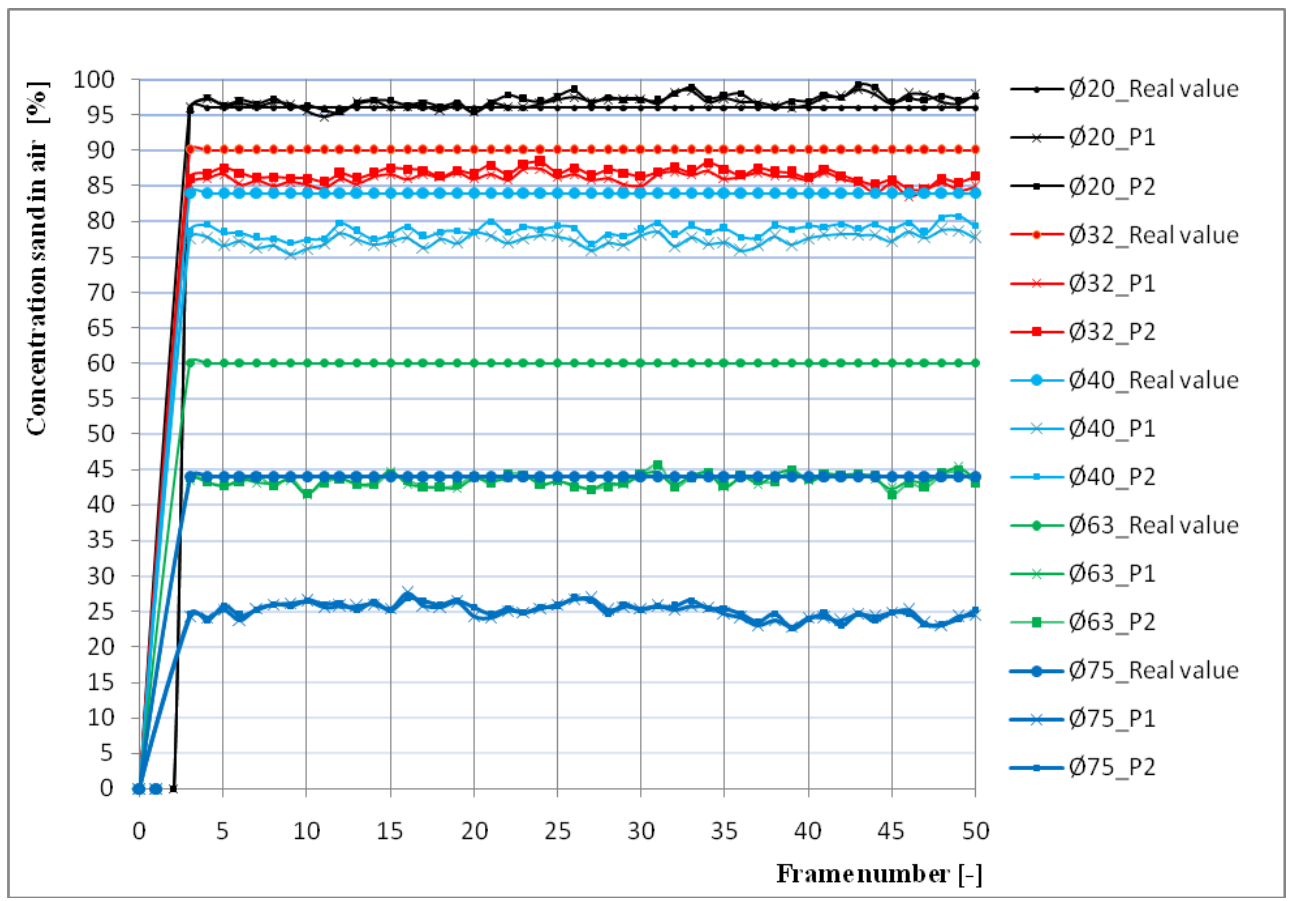

Fig. 6. Concentration of sand and air in cylindrical storage device.

Table 3. Absolute deviation $\Delta \mathrm{x}$.

\begin{tabular}{|c|c|c|c|c|}
\hline Ød [mm] & Plane & $\begin{array}{c}\mathbf{C}_{\mathbf{E X}} \\
{[\mathbf{\%}]}\end{array}$ & $\mathbf{C}_{\mathbf{N}}[\mathbf{\%}]$ & $\Delta_{\mathbf{X}}[\mathbf{\%}]$ \\
\hline \multirow{2}{*}{20} & $\mathrm{P} 1$ & 96 & 96.8 & 0.8 \\
\cline { 2 - 5 } & $\mathrm{P} 2$ & 96 & 97.1 & 1.1 \\
\hline \multirow{2}{*}{32} & $\mathrm{P} 1$ & 90 & 85.9 & 4.1 \\
\cline { 2 - 5 } & $\mathrm{P} 2$ & 90 & 86.7 & 3.3 \\
\hline \multirow{2}{*}{40} & $\mathrm{P} 1$ & 84 & 77.3 & 6.7 \\
\cline { 2 - 5 } & $\mathrm{P} 2$ & 84 & 78.7 & 5.3 \\
\hline \multirow{2}{*}{63} & $\mathrm{P} 1$ & 60 & 43.4 & 16.6 \\
\cline { 2 - 5 } & $\mathrm{P} 2$ & 60 & 43.4 & 16.6 \\
\hline \multirow{2}{*}{75} & $\mathrm{P} 1$ & 44 & 25.0 & 19.0 \\
\cline { 2 - 5 } & $\mathrm{P} 2$ & 44 & 25.1 & 18.9 \\
\hline
\end{tabular}

Absolute deviation for individual tunnels $\Delta \mathrm{X}$ was calculated according to the equation 5 .

$\Delta x=\left\|C_{E X}-C_{\mathbb{N}}\right\|$

\section{Conclusion}


The experimental monitoring of air tunnels within the sand-filled cylindrical storage device demonstrated very positive results. It was discovered that using the capacitance tomography method we are capable of gaining information about the flow disturbances within storage devices during storage processes. In general it is very difficult to get information about processes inside storage devices because it is not possible to use optical measuring equipment. The results obtained show that the $\mathrm{m} 3 \mathrm{c}$ capacitance tomograph can reliably react to changes in the sizes of diameters of air tunnels, as is proven both by the graphic depictions in Table 2 and the curves given in Fig. 6. The resultant average deviations in the concentration of sand correlated with true values are different for each diameter of air tunnel. The smallest deviation is for the smallest diameter of air tunnel Ø20, where the deviation for $\mathrm{P} 1=0.8 \%$ and $\mathrm{P} 2=1.1 \%$. In contrast, the largest deviation was measured for the largest diameter of air tunnel $\varnothing 75$, where the deviation for $\mathrm{P} 1=19 \%$ and $\mathrm{P} 2=18.9 \%$. This experiment was restricted to the monitoring of just one disturbance state, which is an artificially formed air tunnel. In the next phase will performed research of other disturbance states (such as arching over the discharge opening of hoppers or air cavity in the bulk material space) using capacitance tomography.

This paper was conducted within the framework of the project LO1404: Sustainable development of ENET Centre, project SP2018/47: Calibration and experimental devices for the research and validation of simulation models and project SP2018/132: Research and development of innovative transport equipment for verification DEM method in process of bulk solid transportation.

\section{References}

1. J. Zegzulka, Mechanics of bulk materials, (VSB - Technical university of Ostrava, 2004)

2. D. Schulze, Powders and bulk solids: behavior, characterization, storage and flow (New York: Springer, 2008)

3. R. Banasiak, R. Wajman, D. Sankowski, Progress In Electromagnetics Research, 100, , 219-234 (2010)

4. L. Li, J.D. Aubertin, J.S. Dube, The Open Civil Engineering Journal, 8, 1-8 (2014)

5. R. Wajman, R. Banasiak, Ł. Mazurkiewicz, T. Dyakowski, D. Sankowski, Measurement Science and Technology, 17, 8 (2006)

6. M. Soleimani, H. Wang, Y. Li, W. Yang, International journal of information and systems sciences, 3, 2 (2007)

7. S. Ren, F. Dong, Y. Xu, C. Tan, Measurement Science and Technology, 25, 2 (2014)

8. Z. Ye, H.Y. Wei, M. Soleimani, Measurement, 61, 270-279 (2015)

9. T. Dyakowski, L.F.C. Jeanmeure, A.J. Jaworski, Powder Technology, 112, 3 (2000)

10. M. Niedostatkiewicz, J. Tejchman, Z. Chaniecki, K. Grudzie, Chemical Engineering Science, 64, 1 (2009)

11. Ch. Rautenbach, M.C. Melaaen, B.M. Halvorsen, European Journal of Scientific Research, 116, 3 (2013)

12. Y. Li, D.J. Holland, Measurement Science and Technology, 24, 10 (2013)

13. K.Z. Mokhtar, J.M. Saleh, Sensors, 13, 9 (2013)

14. R.A. Williams, S.P. Luke, K.L. Ostrowski, M.A. Bennett, Chemical Engineering Journal, 77, 57-63 (2000)

15. W. Warsito, L.S. Fan, Chemical Engineering and Processing, 42, 663-674 (2003)

16. M. Soleimani, H.G. Wang, Y. Li, W.Q. Yang, International Journal of Information and Systems Sciences, 3, 2 (2007)

17. W.Q. Yang, A.L. Scott, J.C. Gamio, IEEE Transactions on Instrumentation and Measurement, 52, 5 (2003) 
18. M. Sun, S. Liu, Z. Li, J. Lei, Chinese Journal of Chemical Engineering, 16, 4 (2008)

19. J.M. Weber, J.S. Mei, Powder technology, 242, 40-50 (2013)

20. G. Fedorko, P. Liptai, V. Molnar, Eng. Fail. Anal. 83, 30-46 (2018) 